Letter to the Editor

\title{
Nasal congestion with iloperidone therapy in a case series
}

Sir,

Iloperidone or 4'-[3-[4-(6-Fluoro-1,2-benzisoxazol-3-yl)piperidino]propoxy]-3'-methoxyacetophenone is an atypical psychotropic agent belonging to piperidinyl-benzisoxazole derivative, having marked antagonistic actions on adrenergic, serotonergic, and dopaminergic receptors. It is an excellent emerging drug in anxiety and schizophrenia, and particularly useful in acute stages due to involvement of single nucleotide polymorphism in action.

Iloperidone is metabolized primarily by 3 biotransformation pathways: carbonyl reduction, hydroxylation (mediated by CYP2D6) and O-demethylation (mediated by CYP3A4). There are 2 predominant iloperidone metabolites, P95 and P88, having a higher plasma concentration compared with the parent compound. The half lives of these metabolites, drug concentration in plasma and toxicities vary in fast and slow metabolizers of CYP2D6.

Iloperidone is fairly well tolerated. Orthostatic hypotension, somnolence and dizziness are frequently reported apart from fatigue, dry mouth, tachycardia, nasal congestion, hyperprolactinemia, weight gain and hypoglycemia. Other rare adverse effects include QT interval prolongation, leucopenia, neutropenia, agranulocytosis, Neuroleptic Malignant Syndrome (NMS), priapism, suicide and cognitive and motor impairment. However, extra pyramidal symptoms are less. ${ }^{1,2}$

Here we present an interesting case series of iloperidone induced nasal congestion among 23 patients (17-39 years), out of 57 , who were diagnosed schizophrenic and presented in a psychiatry outpatient department in Kolkata, and treated with iloperidone. Initial dose of the drug ranged between 1 and $2 \mathrm{mg}$ twice daily, which were gradually titrated up to $12 \mathrm{mg}$ twice daily. Interestingly, 23 patients presented with intense nasal congestion and headache between 20 and 35 days of taking the escalated dose (Table 1). However, each of them reported to have excellent control of schizophrenic symptoms.

There were no evidences of any co-existing pathological conditions or history of concomitant medications in any patients. Neither of them complained of nasal congestion or headache before initiating iloperidone. Routine blood investigations done in 10 patients were well within normal limits. X-ray of para nasal sinuses done in 11 patients and CT scan of brain done in 3 failed to prove the presence of co-existing sinusitis or other associated pathologies.

For excellent therapeutic responses to schizophrenia, the drug was not withdrawn. Those patients with nasal congestion were symptomatically treated with steam inhalation, oral paracetamol and oxymetazoline nasal drops with very good responses.

Some other side effects of iloperidone were also manifested. 8 patients experienced postural hypotension, 10 had fatigue and dizziness, 4 had dry mouth and somnolence, 4 others had some weight gain, 3 had tachycardia and 2 subjects experienced mild hypoglycemia after taking the drug for at least 4 weeks. But nasal congestion was the most extensive and frequent complaint (23/57 or $40.35 \%)$.

A pooled analysis of 3 double blind, multicenter, placebo and active controlled phase III clinical trials of Potkin et al found that iloperidone was effective in schizophrenia. ${ }^{3}$ A pooled analysis of safety data by Weiden et al from those 3 trials demonstrated the common reported adverse effects of iloperidone as dizzinness, dry mouth, somnolence and dyspepsia. Extra pyramidal symptoms were rather improved with iloperidone. Patients taking iloperidone showed an akathisia profile similar to placebo and weight gain similar to risperidone. QT interval increased significantly but was not associated with significant change in total serum cholesterol. Iloperidone was associated with significant increase in serum glucose and decrease in triglyceride levels. However, prolactin levels were decreased with iloperidone and increased with haloperidol and risperidone. ${ }^{4}$ A randomized, double blind, placebo and active controlled, multicenter 4 -week study by Cutler et al proved the superior efficacy of iloperidone in schizophrenia compared to placebo and ziprasidone. Most adverse events with iloperidone included dizziness, sedation, weight increase, tachycardia, nasal congestion and orthostatic hypotension. Iloperidone was associated with lower rates of sedation, somnolence, extra pyramidal symptoms, akathisia, and restlessness. Significant increases in QT interval occurred in both ziprasidone and iloperidone. Mean changes in total cholesterol, triglycerides, glucose, and prolactin were similar between treatment groups. However, iloperidone was associated with higher rates of weight gain, tachycardia, orthostatic hypotension, dizziness, and nasal congestion. ${ }^{5}$ A pooled analysis from 3 randomized, double blind, multicenter, flexible dose 1 year studies by Kane et al compared iloperidone with haloperidol in schizophrenic patients and found no significant differences in time to relapse between these 2 drugs. The common adverse events with iloperidone were insomnia, nasal

Table 1

Profile of patients who complained of intense nasal congestion and headache on taking iloperidone.

\begin{tabular}{llll}
\hline $\begin{array}{l}\text { Maintenance dose } \\
\text { of iloperidone } \\
\text { (twice daily) }\end{array}$ & $\begin{array}{l}\text { Number } \\
\text { of patients }\end{array}$ & $\begin{array}{l}\text { Range of duration of } \\
\text { initiation of headache } \\
\text { and nasal congestion after } \\
\text { starting the maintenance } \\
\text { dose (days) }\end{array}$ & $\begin{array}{l}\text { Range of period } \\
\text { of recovery on } \\
\text { treatment (days) }\end{array}$ \\
\hline $6 \mathrm{mg}$ & 2 & $29-35$ & $9-10$ \\
$8 \mathrm{mg}$ & 6 & $30-35$ & $4-8$ \\
$10 \mathrm{mg}$ & 7 & $24-33$ & $4-11$ \\
$12 \mathrm{mg}$ & 8 & $21-28$ & $4-8$ \\
\hline
\end{tabular}


congestion, anxiety and tachycardia. Weight gain was greater with iloperidone and changes in QT interval were similar between treatment groups. Iloperidone caused increases in total cholesterol, triglycerides, and glucose levels at the endpoint. ${ }^{6}$

These spontaneous reports of adverse drug reactions are very essential for phase IV trial and global post marketing surveillance for new drugs. The incidence of nasal congestion induced by iloperidone as described here has been exceedingly higher than other reported cases in phase III trials, which might be due to the pharmacogenetic constitution of the population involved. Thus, extensive pharmacogenetic studies are warranted to have an insight on the occurrence of this extensive and singular adverse effect on a specific population like this.

\section{References}

1. Kelleher JP, Centorrino F, Albert MJ, et al. Advances in atypical antipsychotics for the treatment of schizophrenia: new formulations and new agents. CNS Drugs. 2002;16:249-261.

2. Fanapt (Iloperidone) Package Insert. Rockville, MD: Vanda Pharmaceuticals; 2009 [online] http://dailymed.nlm.nih.gov/dailymed/drugInfo.cfm?id=40345 [Revised 03/2011] Accessed 01.03.13.

3. Potkin SG, Litman RE, Torres R, et al. Efficacy of iloperidone in the treatment of schizophrenia: initial phase 3 studies. J Clin Psychopharmacol. 2008;28:S4S11.

4. Weiden PJ, Cutler AJ, Polymeropoulos MH, et al. Safety profile of iloperidone: a pooled analysis of 6-week acute-phase pivitol trials. J Clin Psychopharmacol. 2008;28:S12-S19.

5. Cutler AJ, Kalali AH, Weiden PJ, et al. Four-week, double-blind, placebo- and ziprasidone-controlled trial of iloperidone in patients with acute exacerbations of schizophrenia. J Clin Psychopharmacol. 2008;28:S20-S28.

6. Kane JM, Lauriello J, Laska E, et al. Longterm efficacy and safety of iloperidone: results from 3 clinical trials for the treatment of schizophrenia. J Clin Psychopharmacol. 2008;28:S29-S35.
Saibal Das

Nalmuri Block Primary Health Centre, West Bengal 743 502, India

Indranil Saha

Department of Psychiatry,

Medical College,

Kolkata 700 073, India

Somnath Mondal*

Indian Pharmacopoeia Commission,

Ministry of Health and Family Welfare,

Government of India, Ghaziabad 201 002, India

Department of Clinical and Experimental Pharmacology,

Calcutta School of Tropical Medicine,

Kolkata 700 073, India

Sanjib Bandyopadhyay

Department of Community Medicine, Burdwan Medical College and Hospital,

West Bengal 713 104, India

* Corresponding author. Department of Clinical and Experimental Pharmacology, Calcutta School of Tropical Medicine, 108, CR Avenue, 3rd Floor, Kolkata 700 073, India. Tel.: +91 9874124575 ,

+913322573598.

E-mail addresses: somu1159@gmail.com, somcology@gmail.com

(S. Mondal)

30 June 2013

Available online 7 September 2013 